\title{
The caudate nucleus and acquisition of win-shift radial-maze behavior: Effect of exposure to the reinforcer during maze adaptation
}

\author{
MARK G. PACKARD \\ McGill University, Montreal, Quebec, Canada \\ GORDON WINOCUR \\ Trent University, Peterborough, Ontario, Canada \\ and \\ NORMAN M. WHITE \\ McGill University, Montreah, Quebec, Canada
}

\begin{abstract}
Rats with lesions of the caudate nucleus or sham lesions were trained on a win-shift radialmaze task following one of two types of maze-adaptation trials. In one adaptation condition, rats were allowed to obtain food from maze arms on an unlimited basis for 5 min on 2 consecutive days prior to the start of win-shift food trials. In this maze-adaptation condition, rats with lesions of the caudate nucleus exhibited a transient impairment in the acquisition of win-shift behavior. In a second maze-adaptation condition, different groups of caudate or sham rats were allowed to explore an empty maze for 5 min on 2 consecutive days prior to the start of win-shift food trials. In this maze-adaptation condition, caudate rats were not impaired in the acquisition of win-shift behavior. The role of reinforcers may be one difference in the operating principles that distinguish between caudate-nucleus and hippocampal memory systems.
\end{abstract}

Dissociations between the effects of hippocampalsystem and caudate-nucleus lesions on the acquisition of various learning tasks suggests that these two structures are parts of systems that differ in the types of memory they mediate (Becker, Walker, \& Olton, 1980; Colombo, Davis, \& Volpe, 1989; Cook \& Kesner, 1988; Olton, 1978, 1983; Packard, Hirsh, \& White, 1989; Packard \& White, 1990, 1991). One such dissociation is observed in the performance of win-shift radial-maze behavior following hippocampal or caudate damage. In this task, each of the maze arms is baited with a single food pellet, and optimal performance requires animals to enter each of the baited arms only once on each trial. Animals with hippocampal-system damage are severely impaired in their ability to avoid reentering maze arms in which they have previously obtained reward within a trial (e.g., Becker et al., 1980; Olton, 1978). In contrast, lesions of the caudate nucleus have been reported to have no effect on either the acquisition (Packard et al., 1989; Packard \& White, 1990) or retention (Colombo et al., 1989; Cook \& Kesner, 1988) of win-shift radial-maze behavior. However,

Research was supported by grants from the Medical Research Council of Canada and from FCAR, Province de Quebec, to N.M.W., and by a grant from the Natural Sciences and Engineering Council of Cansda to G.W. Correspondence should be addressed to M. G. Packard, Center for the Neurobiology of Learning and Memory, University of California, Irvine, CA 92717. a transient impairment in the acquisition of win-shift radial-maze behavior following caudate-nucleus damage has also been reported (Winocur, 1980), although the magnitude of the deficit was not as large as that observed following hippocampal damage in the same study and the caudate animals eventually acquired the task. The purpose of the present study was to examine these apparently discrepant findings.

The experiments of Winocur (1980) and Packard et al. (1989) differed in two fundamental ways. First, the size and location of the caudate-nucleus lesions were different. Winocur's lesions were located in the anterior caudate nucleus, and Packard et al.'s lesions were larger, damaging both the anterior and posterior caudate. Second, the procedure used for "adapting" animals to the maze (and maze environment) prior to the start of the win-shift food trials was different in the two studies. In the maze-adaptation procedure of Winocur, rats were placed on the maze and allowed to consume at will food pellets spread over the center platform and arms of the maze. In the maze-adaptation procedure of Packard et al., rats were placed on an empty maze and allowed to explore with no food available.

In a pilot study involving the same rats tested in both laboratories, it was determined that the location and size of the lesions are not the critical factors underlying the different results obtained in the two laboratories. Therefore, the present study was designed to test the hypothesis that the difference in adaptation conditions (i.e., ex- 
posure to a maze containing food vs. an empty maze) was the source of the different effects of caudate lesions on win-shift acquisition. In the maze-adaptation procedure of Winocur (1980), rats were allowed to obtain food from both the center platform and the maze arms. Therefore, the present study included adaptation groups that were allowed to eat pellets from both the maze arms and the center platform or from the center platform only. This comparison was made to determine if the win-shift deficit observed in caudate rats by Winocur was due to experience with obtaining food from the maze arms (which were subsequently baited during win-shift training) or simply due to the presence of food in the maze environment. In addition, this procedural difference may have implications for understanding the operating principles that determine how animals acquire different types of information.

\section{METHOD}

\section{Subjects}

The subjects were 48 male Long-Evans rats (275-300 g). They were housed individually in a temperature-controlled room on a 12:12-h light:dark cycle, with the lights on from 7 a.m. to 7 p.m. All rats were given ad lib access to water.

\section{Apparatus}

The apparatus was a wooden eight-arm radial maze, painted flat gray and elevated $60 \mathrm{~cm}$ above the floor. The maze arms measured $60 \times 9 \mathrm{~cm}$. Food cups were drilled into the floor at the end of each arm. The maze was surrounded by several extramaze cues, including vertical and horizontal striped poster boards, a $25-\mathrm{W}$ lamp, a table, and the seated experimenter.

\section{Surgery \\ Each rat was anesthetized with $60 \mathrm{mg} / \mathrm{kg}$ sodium pentobarbitol, and bilateral electrolytic lesions of the anterior caudate nucleus were made using standard stereotaxic techniques. Stereotaxic coordinates were $\mathrm{AP}=+1.5 \mathrm{~mm}$ from bregma, $\mathrm{ML}= \pm 2.8 \mathrm{~mm}$, and $\mathrm{DV}=$ $-6.2 \mathrm{~mm}$ from the skull surface (Paxinos \& Watson, 1986). Direct current $(4 \mathrm{~mA})$ was passed for $15 \mathrm{sec}$ through an electrode that was insulated except for $0.8 \mathrm{~mm}$ at the tip. Sham-operated rats under- went the identical surgical procedure except that no current was passed through the lowered electrode. The experimental procedure described below began after a 2-week recovery period. During this period, rats with caudate lesions were fed a daily supplement of moistened rat-chow mash.}

\section{Histology}

Following behavioral testing, all rats with lesions were anesthetized with a 1 -cc injection of $30 \%$ chloral hydrate solution and perfused with physiological saline followed by $10 \%$ formol-saline. The rats' brains were removed and fixed in $10 \%$ formol-saline prior to sectioning. The brains were sectioned at $20 \mu \mathrm{m}$ through the lesion area, and every fifth section was mounted and stained using the Kleuver-Berea method (Salthouse, 1964).

\section{Procedure}

Prior to training, all rats were reduced to $85 \%$ of their ad-lib feeding weights by limiting the amount of food available to them and were maintained at this level throughout the testing period. The rats were assigned to one of six groups (three lesion groups and three sham groups, $n=8$ per group) on the basis of the presence or absence of food on the maze during the initial 2-day maze- adaptation period. These included a no-food group, a food-center group, and a food-maze group. The rats in all groups were individually placed on the center platform of the maze and allowed to explore for $5 \mathrm{~min}$ on 2 consecutive days. The rats in the no-food groups were placed on the maze with no food available. For the rats in the food-center groups, 20 pellets (Noyes Original Formula, P. J. Noyes Co., Lancaster, $\mathrm{NH}$ ) were placed on the center platform and none were placed in the arms. For the rats in the food-maze groups, 20 food pellets were placed on the center platform of the maze and 14 pellets were placed on each of the eight arms of the maze, including a single pellet in the food cup.

For all groups, records were kept of the number of arms entered on each of the 2 maze-adaptation days. For the rats in the groups in which food was present on the maze during adaptation, additional records were kept of the following: (1) total number of pellets eaten on the maze arms, (2) total number of pellets eaten on the center platform, (3) total number of maze arms on which food pellets were eaten, and (4) total number of times pellets were eaten on more than one visit to a given maze arm.

Training on win-shift behavior began on Day 3. On these trials, one food pellet was placed in the food cup of each of the eight maze arms and the rats were removed from the maze after the eight pellets had been obtained or $10 \mathrm{~min}$ had elapsed. Records were kept of the arms entered and the order of entry. Visits to unbaited maze arms were scored as errors. Win-shift food trials were run once a day for 10 days.

\section{RESULTS}

\section{Histology}

Results of the histological examination are shown in Figure 1. The lesions produced extensive damage, primarily to the anterior caudate nucleus, ranging from AP 1.5 to $0.1 \mathrm{~mm}$ and ML 1.5 to $3.7 \mathrm{~mm}$ from bregma (Paxinos \& Watson, 1986). Extracaudate damage limited to the overlying cortex was observed in some rats; however, this damage did not correlate with the behavioral results.

\section{Behavior}

Acquisition of win-shift radial-maze behavior for the no-food adaptation groups is shown in Figure 2. A twoway, one repeated measure analysis of variance (ANOVA) computed on the number of errors in the first eight choices revealed no significant group difference $[F(1,14)=1.8$, n.s.]. A significant trial effect showed that all groups improved over the 10 trials $[F(1,9)=9.35, p<.001]$. These results confirm the previous finding that lesions of the caudate nucleus have no effect on the acquisition of win-shift radial maze behavior when no food is present on the maze during the adaptation period (Packard et al., 1989; Packard \& White, 1990).

Acquisition of win-shift behavior for the food-center and food-maze adaptation groups is shown in Figure 3. A two-way, one repeated measure ANOVA computed on the number of errors in the first eight choices revealed a significant group effect $[F(3,28)=4.40, p<.01]$. Tukey's post hoc tests showed that only the caudate foodmaze group was significantly impaired relative to sham animals $(Q=5.69)$. These results confirm the previous finding that exposure to food on the maze arms during the adaptation trials results in a transient impairment in 


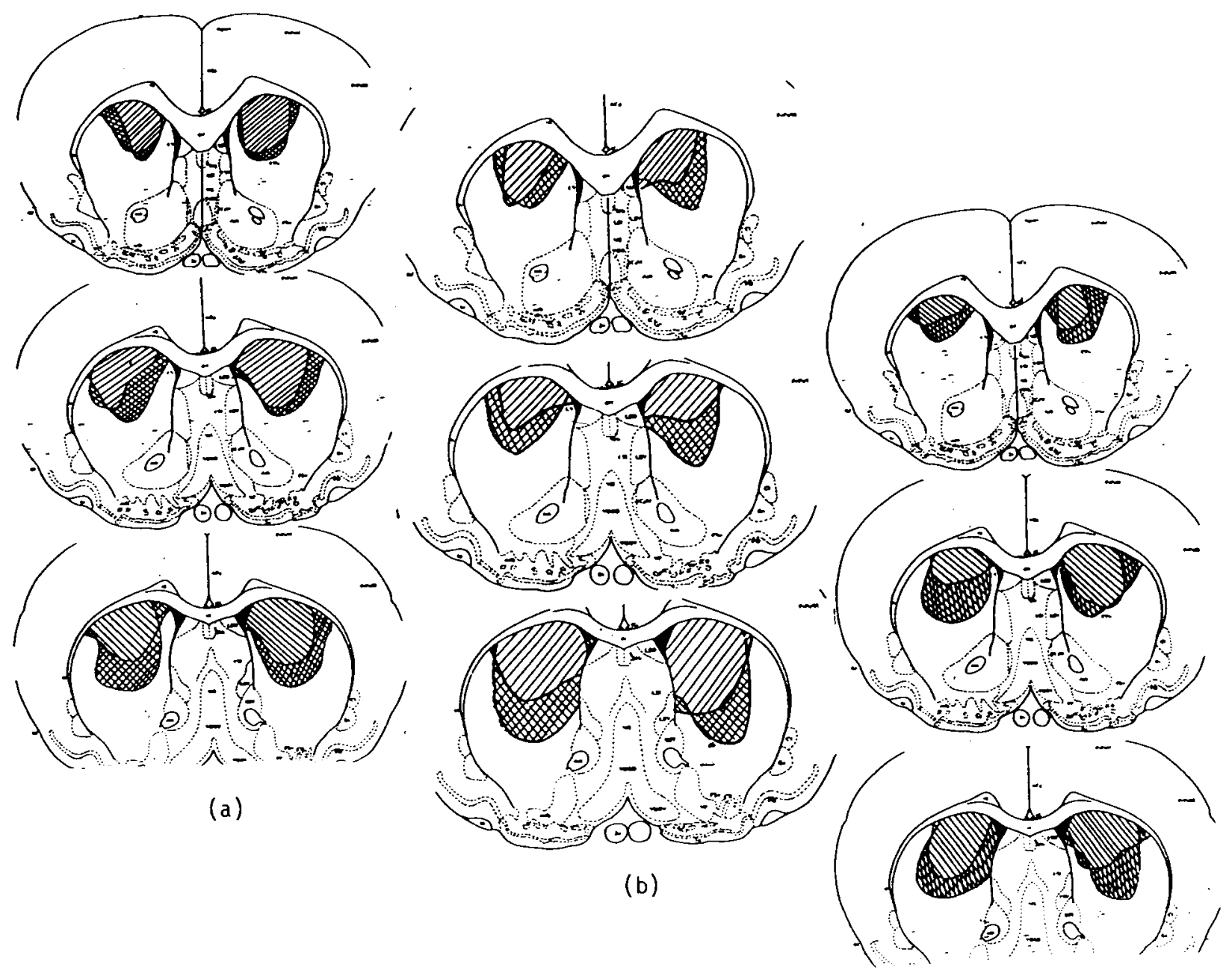

(c)

Figure 1. Illustration of the minimum (single hatched) and maximum (single and double hatched) extent of caudate-nucleus lesions for the (a) food-maze, (b) food-center, and (c) no-food adaptation groups. Lesions are shown at $A P=0.2,0.7$, and $1.2 \mathrm{~mm}$ from bregma. Atlas of Paxinos and Watson (1986).

the acquisition of win-shift behavior (Winocur, 1980). A significant effect of trial $[F(3,9)=6.94, p<.001]$ revealed that win-shift behavior of all groups improved over the 10 trials. This result is also consistent with Winocur's previous finding that the detrimental effect of caudate lesions on win-shift behavior is transient.

It is important to note that exposure to food only on the center platform of the maze had no effect on subsequent acquisition of win-shift behavior, suggesting that the observed deficit was not due to the mere presence of food on the maze during adaptation. The deficit was observed only in the group adapted with food on the maze arms.

The data collected during the adaptation trials are summarized in Table 1. The rats in the three different adap- tation groups did not differ in the mean total number of maze arms visited during the adaptation trials, although the animals in the no-food adaptation groups tended to visit slightly more maze arms on average. This is most likely due to the fact that these rats did not stop exploring the maze in order to eat. There were no differences between the sham groups and their respective lesion groups on any of the other measures used to determine the pattern of eating of the rats in the food-center or food-maze groups (i.e., mean number of pellets eaten on the maze arms, mean number of pellets eaten on the center platform, mean number of maze arms on which food pellets were eaten, and mean number of times pellets were eaten on more than one visit to a given maze arm). These findings suggest that the pattern of eating on food-center or 


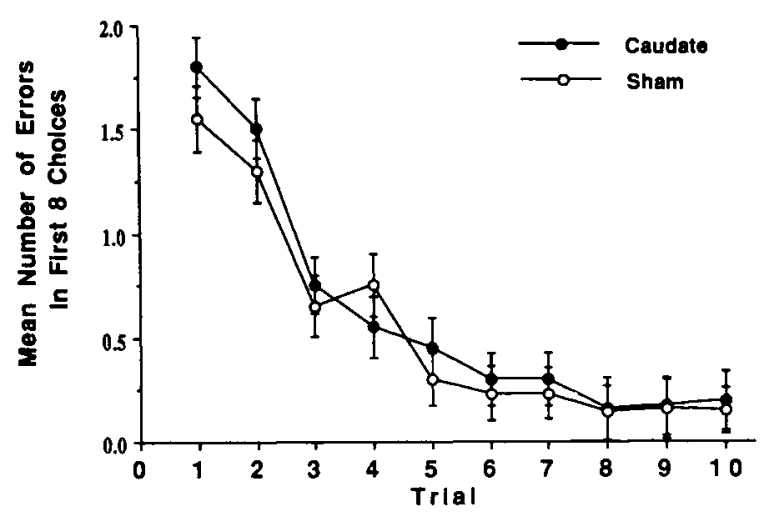

Figure 2. Mean number of errors in the first eight choices for the no-food adaptation groups. Vertical bars represent standard error of the mean.

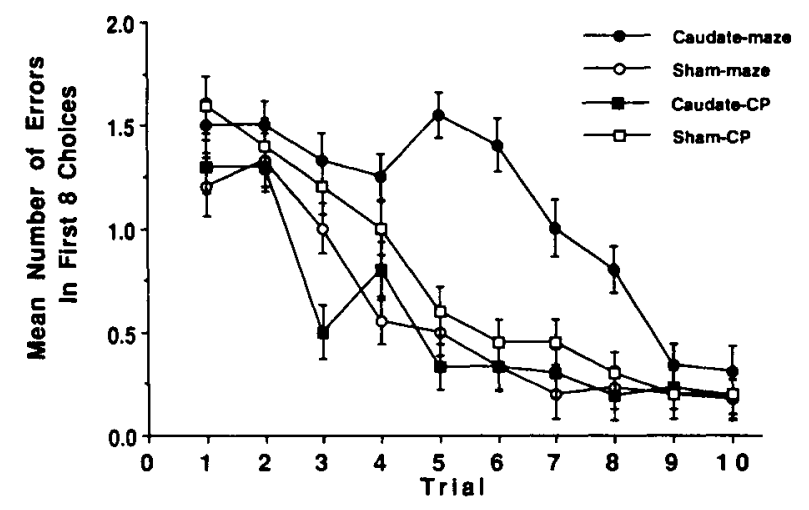

Figure 3. Mean number of errors in the first eight choices for the food-maze and food-center adaptation groups. CP = center platform. Vertical bars represent standard error of the mean.

food-maze adaptation trials by caudate and sham rats was not responsible for subsequent differences in the acquisition of win-shift radial-maze behavior.

\section{Discussion}

The results of the present study suggest that the tendency of caudate-nucleus lesions to produce a transient impairment in the acquisition of win-shift radial-maze behavior is related to exposure to the reinforcer on the arms of the maze during adaptation to the maze environment prior to the start of win-shift trials. When rats with caudate lesions were allowed to consume food in the arms of the maze during the initial 2-day adaptation period, a transient deficit in the acquisition of win-shift behavior was observed. In contrast, rats with caudate lesions, allowed to explore an empty maze during adaptation or to eat only on the center platform, showed no deficit. These findings suggest that the difference in maze-adaptation procedures used by Winocur (1980) and Packard et al. (1989) was the source of the different results reported in the two studies. Specifically, the present findings suggest that consumption of the reinforcer on the maze arms during adaptation was responsible for the behavioral deficit observed in the rats with caudate lesions.

An explanation of this pattern of results can begin with a consideration of two observations. First, opposite behavioral contingencies were reinforced during the adaptation and win-shift phases of the experiment. During adaptation, the rats could and often did obtain food from a given maze arm more than once. Therefore, a tendency to repeat visits to maze arms was reinforced during these trials, a contingency that can be described as "win-stay." In contrast, during the subsequent win-shift food trials, the rats had to learn to enter each of the baited arms only once on each trial and to avoid revisiting maze arms in which a reinforcer had been obtained, a contingency that has been described as "win-shift." The possibility for interference when win-shift training follows immediately after 2 days of win-stay adaptation are obvious.

The second observation is that this interference was not observed in normal rats, but only in rats with caudate lesions. This suggests that the neural system of which the caudate nucleus is a part gave normal rats the ability to acquire a win-shift contingency immediately after experience with the win-stay contingency, without interference. A more general form of this observation has been made by several other investigators, who have suggested that the caudate nucleus mediates the acquisition of "alternative strategies" required for learning (Whishaw, Mittleman, Bunch, \& Dunnett, 1987; see also Jaspers, de Vries, \& Cools, 1990a, 1990b).

The roles of the caudate nucleus and of reinforcer contingencies in memory have been the subjects of other recent experiments and theorizing, and these are of value in understanding the present observations. A number of workers have suggested that the brain contains more than one more-or-less-independent memory system, each of which acquires a different type of information (e.g., Hirsh, 1974; Mishkin \& Petrie, 1984; O'Keefe \& Nadel, 1978; Olton, Becker, \& Handelmann, 1979; Sutherland \& Rudy, 1989). Mishkin, Malamut and Bachevalier (1984) proposed a dual-memory theory that draws a broad distinction between a "cognitive" memory system subserved in part by the hippocampus and a stimulusresponse (S-R) or "habit" system, which is thought to be mediated by the caudate nucleus and its cortical afferents. It has also been suggested that reinforcers play different roles in these hypothetical memory systems (Mishkin \& Petri, 1984; Packard et al., 1989; Packard \& White, 1990). Within the hippocampal cognitive system, animals acquire information about the stimulus properties of reinforcers in relation to other stimuli, in the manner proposed by cognitive-learning theory (e.g., Tolman, 1932 , 1948). This process of learning about reinforcers is thought to occur in the win-shift task. Within the caudate habit system, repeated exposure to the same reinforcer contingency incrementally strengthens associations between stimuli and responses, in the manner proposed by S-R learning theory (e.g., Hull, 1943; Thorndike, 1933). This action of reinforcers is thought to occur in 
Table 1

Behavior During Adaptation

\begin{tabular}{|c|c|c|c|c|c|c|}
\hline & \multirow[b]{2}{*}{ Caudate } & \multirow[b]{2}{*}{ Sham } & \multicolumn{2}{|c|}{ Food Maze } & \multicolumn{2}{|c|}{ Food Center } \\
\hline & & & Caudate & Sham & Caudate & Sham \\
\hline \multicolumn{7}{|c|}{ Food Adaptation Groups } \\
\hline \multicolumn{7}{|l|}{ Mean number of: } \\
\hline pellets eaten on the maze arms & 25.2 & 28.3 & & & & \\
\hline $\begin{array}{l}\text { maze arms on which food pellets were eaten } \\
\text { times in which pellets were eaten on }\end{array}$ & 8.7 & 7.9 & & & & \\
\hline more than one visit to a given maze arm & 4.2 & 3.7 & & & & \\
\hline pellets eaten on center platform & & & 18.5 & 19.0 & 17.7 & 19.5 \\
\hline maze arms visited & & & 9.1 & 8.2 & 7.6 & 9.3 \\
\hline \multicolumn{7}{|c|}{ No-Food Adaptation Groups } \\
\hline maze arms visited & 11.6 & 10.5 & & & & \\
\hline
\end{tabular}

certain win-stay tasks, such as those involving simultaneous discriminations.

The results reported by Packard et al. (1989) are consistent with these ideas. Rats with caudate-nucleus lesions were impaired on acquisition of a win-stay task, and animals with hippocampal-system (fornix) lesions were impaired on acquisition of the win-shift task. This suggests that normal animals may acquire win-stay behavior with a neural mechanism that includes the caudate nucleus and that they may acquire win-shift behavior with a neural mechanism that includes the hippocampus. In the present experiment, it may be that this separation of functions in the normal rats allowed them to acquire opposing contingencies without interference.

To explain the deficit observed in the rats with caudatenucleus lesions, we can hypothesize that the remaining tissue in these rats, including the hippocampus, acquired both reinforcement contingencies. When the win-shift trials started, interference from the presence of previously acquired win-stay information may have occurred, accounting for the performance deficit (or difficulty in switching strategies) observed.

This hypothesis may appear to contradict a previous finding (Packard et al., 1989) that caudate animals were unable to learn a win-stay task in an eight-arm radial maze. However, because of a difference in the win-stay procedures in the two experiments, this contradiction is more apparent than real. In the present experiment, the win-stay contingency could be learned in two different ways, both of which would produce the same win-stay behavior. In the caudate (S-R) system, the win-stay contingency could be represented as a strengthened (reinforced) tendency to approach all arms. In the hippocampal (cognitive) system, the same win-stay contingency could be represented as the information that all arms contain food at all times. In the rats with caudate lesions, the acquisition of this information by the hippocampal system during adaptation may have interfered temporarily with subsequent learning of win-shift information when that contingency was introduced.

In our previous win-stay experiment (Packard et al., 1989), animals found food in a different set of four illuminated maze arms each day. In this situation, the win-stay contingency could be represented in the caudate S-R system as a strengthened tendency to approach lit arms. However, this task is problematic for the hippocampal system because the location of food with respect to most stimuli in the maze environment is inconsistent. Any information acquired about the relationship of the food to such stimuli would fail to produce accurate performance. This is the result that was observed in animals with caudate-nucleus lesions. It should be noted that although there is no a priori reason why the hippocampal system should be unable to represent the consistent relationship of the food to the lights even though both are on different arms each day, this does not seem to happen: animals with caudate lesions did not show any sign of acquiring this "pure" win-stay contingency after 15 days of testing (Packard et al., 1989). This may be because the lights are on the maze itself, rather than distal to it, and/or because their locations with respect to other stimuli change each day.

This hypothesis is also consistent with the observation of Packard et al. (1989) that acquisition of win-stay behavior was enhanced by hippocampal-system lesions. Similar observations have been reported by others (e.g., Eichenbaum, Fagan, Matthews, \& Cohen, 1988; Stevens \& Cowey, 1972). In learning situations that happen to match the capability of a nonhippocampal memory system, removing the hippocampus may eliminate interference and leave the nonhippocampal system free to acquire and express the memory more quickly.

The remaining question concerns the normal rats in the present experiment. Why didn't their hippocampi acquire the cognitive-type win-stay information, just as it did in the case of the rats with caudate lesions? It can be hypothesized that the acquisition of the win-stay contingency by the caudate system in normal rats either inhibits the same type of learning by the hippocampal system or is somehow registered by the hippocampus. Both of these possibilities would leave the hippocampal system free to acquire other behaviors, such as win-shift, in an unimpeded manner.

Taken together, these findings suggest that the conditions of an animal's initial exposure to a situation may be important in determining what is learned about the sit- 
uation, how it is learned, and where in the brain it is stored. The present results also raise numerous questions about the interactions among the various brain systems involved in learning and memory. The factors affecting these processes are poorly understood, and their elucidation will require much further work.

\section{REFERENCES}

Becker, J. T., Walker, J. A., Olton, D. S. (1980). Neuroanatomical basis of spatial memory. Brain Research, 200, 307-320.

Colombo, P. J., DAvis, H. P., \& VolPE, B. T. (1989). Allocentric spatial and tactile memory impairments in rats with dorsal caudate lesions are affected by preoperative training. Behavioral Neuroscience, $103,1242-1250$.

CoOK. D., \& KeSNer, R. P. (1988). Caudate nucleus and memory for egocentric localization. Behavioral \& Neural Biology, 49, 332-343.

Eichendaum, H., Fagan, A., Matthews, P., Cohen, N. J. (1988). Hippocampal system dysfunction and odor discimination learning in rats: Impairment or facilitation depending on representational demands. Behavioral Neuroscience, 102, 331-339.

HIRSH, R. (1974). The hippocampus and contextual retrieval of information from memory: A theory. Behavioral Biology, 12, 421-444.

HulL, C. L. (1943). Principles of behavior. New York: AppletonCentury-Crofts.

JASPers, R. M. A., DE VRIES, T. J., \& Cools, A. R. (1990a). Effects of intrastriatal apomorphine on changes in switching behaviour in duced by the glutamate agonist AMPA injected into the cat caudate nucleus. Behavioral Brain Research, 37, 247-254.

Jaspers, R. M. A., DE Vries, T. J., \& Cools, A. R. (1990b). Enhancement in switching motor patterns following local application of the glutamate agonist AMPA into the cat caudate nucleus. Behavioral Brain Research, 37, 237-246.

Mishkin, M., Malamut, B., \& Bachevalier, J. (1984). Memories and habits: Two neural systems. In G. Lynch, J. L. McGaugh, \& N. M. Weinberger (Eds.), Neurobiology of leaming and memory (pp. 65-77). New York: Guilford.

Mishkin, M., Petri, H. L. (1984). Memories and habits: Some implications for the analysis of learning and retention. In N. Butters \& L. R. Squire (Eds.), Neuropsychology of memory (pp. 287-296). New York: Guilford

O'KeEFE, J., \& NADEL, L. (1978). The hippocampus as a cognitive map. Oxford: Oxford University Press.
Olton, D. S. (1978). The function of septohippocampal connections in spatially organized behavior. Functions of the septo-hippocampal system, Ciba Foundation Symposium, 58, 327-342.

OLton, D. S. (1983). Memory functions and the hippocampus. In W. Seifert (Ed.), Neurobiology of the hippocampus (pp. 335-372). New York: Academic Press.

Olton, D. S., Becker, J. T., Handelmann, G. E. (1979). Hippocampus, space, and memory. Behavioral \& Brain Sciences, 2 , 313-365.

Packard, M. G., Hirsh, R., White, N. M. (1989). Differential effects of fornix and caudate nucleus lesions on two radial maze tasks: Evidence for multiple memory systems. Journal of Neuroscience, 9, 1465-1472.

PACKard, M. G., \& White, N. M. (1990). Lesions of the caudate nucleus selectively impair acquisition of "reference memory" in the radial maze. Behavioral \& Neural Biology, 53, 39-50.

PACKARD, M. G., * Whrte, N. M. (1991). Dissociation of hippocampus and caudate nucleus memory systems by posttraining intracerebral injection of dopamine agonists. Behavioral Neuroscience, 105, 73-84.

Paxinos, G. WAtson, C. (1986). The rat brain in stereotaxic coordinates. New York: Academic Press.

SAlthouse, T. N. (1964). Luxol fast blue " G" as a myelin stain. Stain Technology, 39, 123-128.

Stevens, R., Cowey, A. (1972). Enhanced alternation learning in hippocampectomized rats by means of added light cues. Brain Research, 46, 1-22.

Sutherland, R. J., Rudy, J. W. (1989). Configural association theory: The role of hippocampal formation in learning, memory, and amnesia. Psychobiology, 17, 129-144.

THORNDIKE, E. I. (1933). A proof of the law of effect. Science, 17, 173-175.

Tolman, E. C. (1932). Purposive behavior in animals and men. New York: Appleton-Century-Crofts.

Tolman, E. C. (1948). Cognitive maps in rats and men. Psychological Review, 56, 144-155.

Whishaw, I. Q., Mittleman, G., Bunch, S. T., \& Dunnett, S. B. (1987). Impairments in the acquisition, retention, and selection of spatial navigation strategies after medial caudate-putamen lesions in rats. Behavioral Brain Research, 24, 125-138.

Winocur, G. (1980). The hippocampus and cue utilization. Physiological Psychology, 8, 280-288.

(Manuscript received August 27, 1991; revision accepted for publication February 28, 1992.) 